\title{
DYT1 dystonia-associated mutant affects cytoskeletal dynamics
}

\author{
Santos, M. , Rebelo, S. , da Cruz e Silva, E. F. , da Cruz e Silva, O. A. B. \\ *Center for Cell Biology, Health Sciences and Biology Department, University of Aveiro, Campus de \\ Santiago, 3810-193 Aveiro, Portugal
}

Email: marianasmg@gmail.com

TorsinA and its related family members belong to the ATPases associated with a variety of cellular activities $\left(\mathrm{AAA}^{+}\right)$superfamily. TorsinA has been the most studied torsin family member due to its association with DYT1 dystonia. A mutation of a single glutamic acid within the C-terminal of torsinA $(\Delta \mathrm{E} 302 / 303$, also referred to as $\Delta \mathrm{E}$ ) was found in most cases of DTY1 dystonia [1]. TorsinA is primarily located in the endoplasmic reticulum (ER) lumen and nuclear envelope (NE), while $\Delta \mathrm{E}$-torsinA is abnormally concentrated in the NE [2]. TorsinA has been implicated in cytoskeleton dynamics through interaction with different binding partners. However, it seems that the $\Delta \mathrm{E}$-torsinA mutation interferes with cytoskeleton dynamics [3, 4]. Therefore, we analyzed cytoskeleton alterations associated with the $\Delta$ E-torsin $A$ mutation. In order to achieve this goal, we transfected SH-SY5Y cells with GFP-wt-torsinA or GFP- $\Delta$ E-torsinA and performed immunofluorescence analysis with $\beta$-tubulin and acetylated $\alpha$-tubulin specific antibodies and with phalloidin that binds to F-actin. We showed that in wt-torsinA transfected cells, $\beta$-tubulin, acetylated $\alpha$-tubulin and Factin were distributed throughout the cytoplasm, in a manner similar to non-transfected cells (Fig. 1A). In contrast, in some $\Delta \mathrm{E}$-torsinA transfected cells, the distribution of those markers is altered, being more restricted to the NE, and cells seem to be less intensely labeled. Moreover, in some cells, $\beta$-tubulin colocalized with $\Delta \mathrm{E}$-torsin $\mathrm{A}$ positive inclusions (Fig. 1A). Further, when we quantified the fluorescence intensity (FI) of $\beta$-tubulin, we detected a slight decrease of the FI in $\Delta \mathrm{E}$-torsinA transfected cells compared to wt-torsinA transfected cells (Fig.1B). In the same way, $\Delta$ E-torsinA transfected cells have lower levels of acetylated $\alpha$-tubulin, which is a marker for microtubules stability, suggesting that microtubule dynamics may be compromised by the $\Delta \mathrm{E}$-torsinA mutation. Furthermore, we observed a loss of F-actin stress fibers in $\Delta \mathrm{E}$ torsinA transfected cells (Fig. 1A). Indeed, we showed that F-actin FI decrease from 1.03 to 0.83 (around $20 \%$ ) in $\Delta \mathrm{E}$-torsinA transfected cells compared with wt-torsinA transfected cells (Fig. 1B). Our results are in agreement with previous reports where it was reported that the expression of $\Delta \mathrm{E}$-torsin $\mathrm{A}$ altered the localization of vimentin to the NE [3], KLC to inclusions and nesprin-3 to the ER [4]. Nesprins in the outer nuclear membrane bind to actin, microtubules and intermediate filaments, thus forming a complex that links the nucleoskeleton and cytoskeleton (the LINC complex). Therefore, torsinA and its binding partners may have a role in modulating the LINC complex. Disruption of the LINC complex may contribute to the development of muscular dystrophies and cardiomyopathies.

We thank Dr. William Dauer (University of Michigan) for providing torsinA plasmids. This work was supported by the FCT, CBC - University of Aveiro, REEQ/1023/BIO/2005, Pest-OE/SAU/UI0482/2013, PTDC/BEX-BCM/0493/2012, JPND-BIOMARKAPD and the scholarship awarded to MS (SFRH/BD/65353/2009).

[1] Ozelius, L.J. et al, Nat Genet, 17, 40-48, 1997.

[2] Goodchild, R.E. and Dauer, W.T., Proc Natl Acad Sci USA, 101, 847-852, 2004.

[3] Hewett, J.W. et al, Neurobiol Dis, 22, 98-111, 2006. 
[4] Nery, F.C. et al, J Cell Sci, 121, 3476-3486, 2008.

[5] Santos, M., et al, Microsc Microanal, 18, 41-42, 2012.

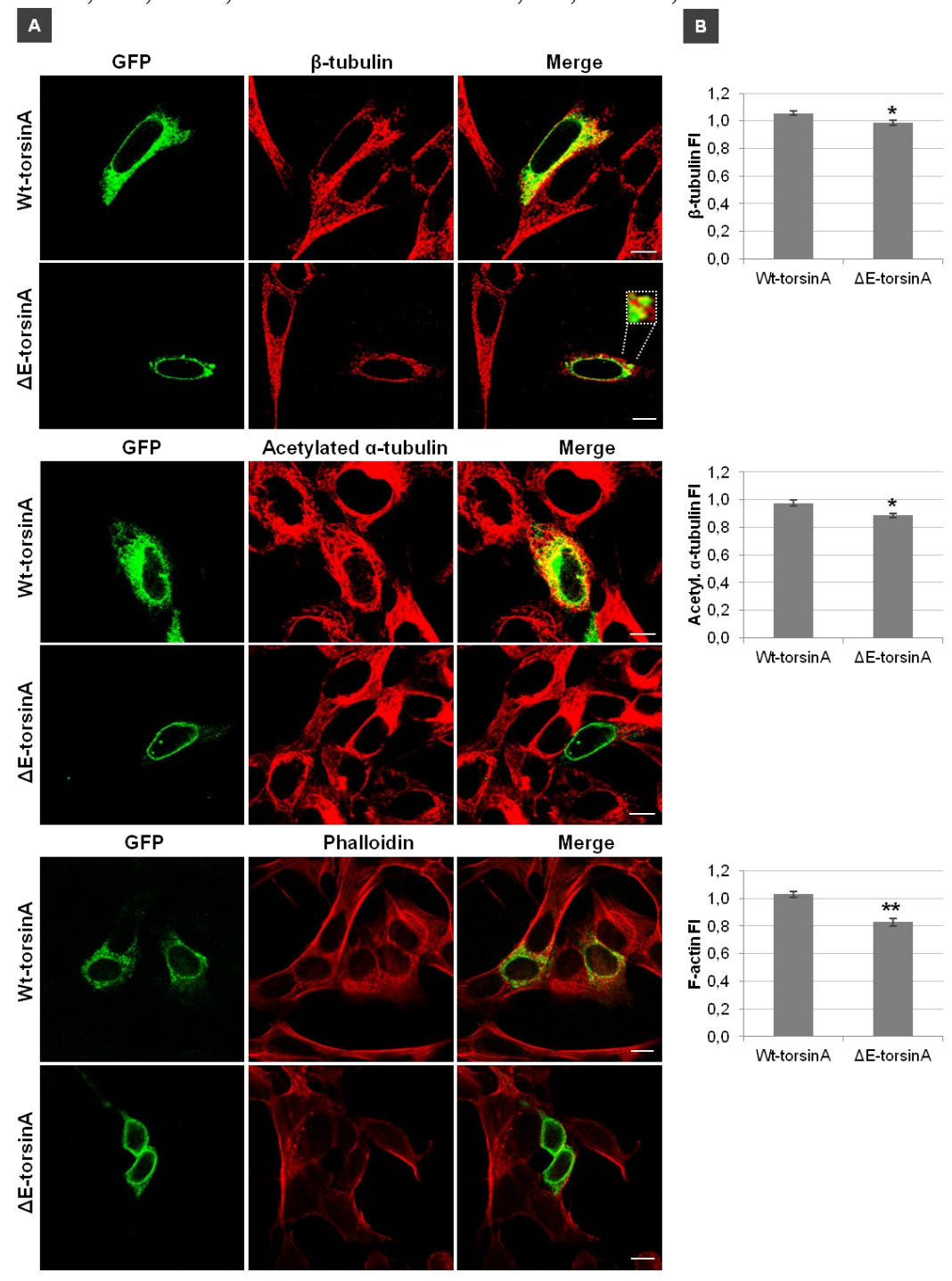

Figure 1. Distribution of $\beta$-tubulin, acetylated $\alpha$-tubulin and $F$-actin in wt- and $\Delta E$-torsin $A$ transfected cells. A- SH-SY5Y cells were transfected with GFP-wt-torsinA or GFP- $\Delta$ E-torsinA. Specific primary antibodies for endogenous $\beta$-tubulin and acetylated $\alpha$-tubulin were detected with Alexa Fluor 594-conjugated secondary antibody (red). Alexa Fluor 594-conjugated phalloidin (red) was used to label F-actin. The higher magnification view shows in more detail the co-localization of $\Delta \mathrm{E}$-torsin $\mathrm{A}$ and $\beta$-tubulin in inclusions. B- Quantification of $\beta$ tubulin, acetylated $\alpha$-tubulin (acetyl. $\alpha$-tubulin) and F-actin fluorescence intensity (FI), which represents the ratio transfected cells FI/total cells FI, in wt- and $\Delta$ E-torsinA transfected cells. Values are mean \pm SEM, $n=22$ cells (for $\beta$-tubulin), 30 cells (for acetyl. $\alpha$-tubulin) and 20 cells (for F-actin). Statistical significance analysis was conducted by Student's t-test. Statistically different from wt-torsinA transfected cells, $* \mathrm{p}<0.05, * * \mathrm{p}<0.01$. Photographs were acquired using a LSM 510-Meta confocal microscope [5]. The argon laser line of $488 \mathrm{~nm}$ and the $561 \mathrm{~nm}$ DPSS laser were used. Microphotographs were acquired in a sole section in the Z-axis (xy mode) and represent a mean of 16 scans. Profiles were acquired using the Zeiss LSM 5104.0 software. Bars, $10 \mu \mathrm{m}$. 